www.conferenceie.ase.ro

\title{
IOT SECURITY, ASSOCIATED MALWARE TRENDS AND VULNERABILITIES
}

\author{
Sttefan NICULA \\ Bucharest University of Economic Studies \\ niculastefan13@stud.ase.ro \\ Răzvan Daniel ZOTA \\ Bucharest University of Economic Studies \\ zota@ase.ro
}

\begin{abstract}
The research analysis conducted on various IoT devices revealed security issues with patterns that are strongly related to the limitations in terms of implementation. This includes the technology-stack used, hardware capabilities and missing security best practices and standards. This paper tackles multiple vectors of attack that are threatening the privacy and security integrity level of IoT devices. Moreover, the research perspective covers the malware incident aspect, vulnerabilities that are affecting different components and the overall security level provided by the products. Given the relative early stages of IoT product development, comparison between the early and the current stage of web and mobile development phases with the IoT area displays certain similarities. Malware outbreaks are studied along with the impact of publicly known vulnerabilities, the attack surface of an IoT device and the mitigations enforced by some vendors. The security evaluation methodology was based on Penetration Testing practices, targeting all the components exposed by the IoT devices that were studied. This included the network capabilities, web and mobile applications and targeted the physical attack vectors as well.
\end{abstract}

Keywords: IoT, security, vulnerabilities, malware

JEL classification: L86

DOI: $10.24818 / \mathrm{ie} 2020.01 .05$

\section{Introduction}

The paper aims to reveal, categorize and interpret vulnerabilities and privacy concerns across a series of studied IoT devices. The study has been conducted on a number of research projects across a multi-purposes area design for IoT devices.

The IoT security analysis was from a Penetration Testing perspective in order to conduct various research exercises to obtain metrics which can later be used as base references for the conclusions [1].

Such projects often had complex architecture designs because the intended purpose of the tested IoT solutions was to provide an automated and independent solution to publicly known problems. Based on these principles, the tested IoT products had multiple components that needed to be analyzed. Following the business logic of the tested products, the scope of the project included different technical components such as web applications, mobile applications and hardware physical devices that were involved in the Penetration Testing engagement and were taken as part of the main scope [2].

From previous past experiences, these systems defined as a single IoT product, have unique vulnerabilities that can affect the studied IoT product in different ways. However, these 
www.conferenceie.ase.ro

vulnerabilities can be noticed across the entire spectrum covered by the IoT product in their business area.

\section{The research base}

The following list contains the most important IoT products in terms of relevance that were tested as part of this research:

1. Robot device with camera, Wi-Fi capabilities and speakers (an IoT product containing a robot made from hardware pieces, a mobile application that can be linked with the robot via direct Wi-Fi connection, a web application hosted on the robot motherboard and exposed via port 80, a terminal CLI port exposed by the robot and a desktop application that communicates via a predefined port number) [3];

2. Smart gas station (tested a gas station that implements smart payment systems connected locally to multiple servers, the scope also included a card reader, a payment terminal and a point of sale machine defined as a sandbox touch-screen display hosting a Windows system);

3. Fingerprint payment solution (the scope was defined as a set of fingerprint readers implemented in conjunction with a retail payment terminal, a web application linked all the fingerprints and made all the connections);

4. Tire pressure monitoring system (a smart pressure valve inserted inside tires from vendors, using Bluetooth connection to communicate with a custom mobile application);

5. ATM machines (tested multiple ATM machines as part of stand-alone system and payment terminals; testing included lockpicking, security case tests, operating system tests and physical components test like keyboard, touchscreen and hard-drive);

6. Various routers (analyzed internet routers from multiple vendors).

From the provided research projects, some of the most common vulnerabilities that were identified can be categorized into:

1. Missing authorization and authentication (one of the most common and high risk vulnerabilities identified across the products studied is the lack of authorization for important and critical functionalities such as administration interfaces; cases of default credentials have been identified and exploited, multiple important functionalities such as CLI terminals accessible without any authentication in-place);

2. Injection types of attacks (vulnerabilities such as Cross-Site Scripting, SQL Injection, Remote Code Execution and others have been identified, specifically for products that have embedded web application such as routers and IoT robots; these vulnerabilities are being reproduced due to missing user supplied-input validation and sanitization for many of the inputs exposed by the web applications);

3. Business logic flaws (even though adequate security controls were implemented for web applications, mobile interfaces and other components, the business flow could be exploited in order to cause a high impact on the affected functionalities);

4. Sensitive information disclosure (identifying cases of sensitive information disclosure such as payment details, personal identification information, card details, personal health details, all of them accessible in an unauthenticated or unauthorized manner);

5. Android and iOS mobile applications lacking security best practices (no root detection implemented, missing SSL Pinning mechanisms, source code not obfuscated, exported interfaces without proper external interaction validation; all these practices that were not implemented made room for attack vectors to be identified due to the ease of analyzing the mobile application and its internals); 
www.conferenceie.ase.ro

The methodology for testing the presented IoT products was that of a Penetration Testing engagement, targeting all the components from the network layer perspective, to application layer and included the physical aspect as well.

\section{Vulnerabilities and malware incidents}

An attack chain for a compromised IoT device can usually be linked to patterns that have similar root causes. Often times, we see attacks that gain initial access using low-hanging fruits like default passwords or the ones mentioned in the previous chapter. The term low-hanging fruits is most of the time associated with vulnerabilities that are easy to find and easy to exploit. The category can start from missing access controls to injection types of attacks and exposed control ports.

Using the initial foothold obtained, the attackers are leveraging vulnerabilities to elevate the privileges obtained inside the system. Sometimes, attackers do not even require a system level access in order to abuse the device. This is the case of botnets or spywares where IoT products are "incorporated" into the attacker's network of infected products and used, for example, as DDoS machines, as a pivot system inside a local network or a proxy server.

Some common features regarding IoT products such as routers are related to exposed VPN tunneling. This is an important aspect as VPN solutions are usually safe, especially the ones that have strong implementations of open-source, up to date, mature VPN solutions. However, outdated software do have vulnerabilities that are publicly known and exploited in the wild. As such, these solutions deployed on specific IoT devices can expose an entire product range [4]. There is also the risk of a product being affected by a publicly available vulnerability [5]. In this case the risk is greater as mass-scanning can be launched across the internet in order to find exposed devices. There are also online services that are active as databases for quickly identifying ports exposed on the internet [6].

By studying popular malware outbreaks, we can make a direct correlation between important, wide-spread CVEs disclosed for popular brands and malware campaigns [7].

An important aspect regarding IoT malware campaigns is the initial payload delivery method. Comparing the that with a first stage malware chain for Windows victims, the IoT product requires the attacker's direct contact with the device. Most of the times, the victim will not have direct access to the IoT product compared to the access of a Windows operating system. Attacks such as phishing or accidental malware execution like Trojans, are not applicable.

There are also concerns regarding data privacy and manipulation or the threat of a backdoor in the product. These concerns are very dependent on the devices' capability of collecting, storing and processing sensitive data. Even though they are not the topic covered in this article, it's worth mentioning that such security flaws can sometimes be exploited by other malicious actors as well. If backdoors are uncovered and exposed or certain privacy risks are being made public, the affected products can become a high-value target to attackers, increasing the risk of being compromised [8].

In terms of research and responsible vulnerability disclosure, we can identify some patterns that are similar to web application development in the early stages of public disclosures. Due to the initial stages of the IoT products, there are but a small number of mature vendors that are enlisting as publicly available for research and testing. This is a big step for a company, as public enrolling can bring great benefits to the overall coverage but it also requires a certain level of maturity and development. Big companies are always having a bigger advancement level in terms of security and this can be correlated with the allocated budget as well [9]. 


\section{Comparison between IoT, web and mobile}

The discrepancy between the IoT and other areas such as web development and mobile can be linked with the lack of frameworks, regulations and technology-stack patterns that are currently missing from the IoT area or are underdeveloped. We can compare web development or mobile development in this regard. Currently, there are very mature solutions for webservers, backend and client-side frameworks. We can see a lot of workarounds and limitations when it comes to IoT products. The technologies implemented are relatively primitive to other counterparts in the same area, like webservers deployed on IoT devices and on internet-facing servers.

However, those limitations or workarounds that are implemented are related, on a strong proportion, to hardware specifications. Robust and mature frameworks or operating systems do require more computing power in order to be used at full capacity. This is often an important limitation in an IoT product although we can see improvements in terms of hardware specifications as well [10]. As technology evolves, so are the IoT devices. With more computing power, storage and improvements related to memory, there is also a bigger security level enforced on the devices.

Going back to the comparison between web development and the IoT area in terms of security. In the initial stages of web development, a lot of easy to find vulnerabilities were found and reported or abused in the wild. The level of difficulty required to identify and exploit one was exponentially lower compared to today's practice. This can also be seen in the annual reports of public bug bounty platforms [11] and the increased number of targets versus the reported vulnerabilities overall. Of course, when speaking of payment per vulnerability reported, the revenue is also much bigger than previous years but that reflects the level of maturity that the web area has improved in terms of security.

We can expect this from the IoT perspective as well in the next years as the trend was translated in the mobile area too although this can be argued because the mobile area has major big companies that are regulating or controlling important aspects security-related like the development platform, application deployment, operating systems and privacy restrictions.

Some vendors have already started to implement local third party software to embed inside their products for an increased resistance against outside threat [12]. Antiviruses offer a great protection in terms of malware. Although they don't act like an IPS or IDS network monitoring tool, they do provide protection against publicly known threats, identify malware, malicious behavior and determine potentially compromised devices. This alternative has benefits in terms of size and computing power for the third party software that is deployed on the IoT device. It is not meant to offer the same protection as dedicated security devices placed on the network level but it offers a protection alternative installed directly on the IoT device that is adapted to the product's hardware limitations.

\section{Conclusions}

The IoT security area represents the security of a larger spectrum of technologies combined into a standalone product. Hence, the attack surface can be significant in width while also limited in terms of architectural depthness and complexity. Hardware capabilities and their limitations play an important role in the product development maturity level. As multiple frameworks and technology stacks are emerging, so are the standards for security best practices and implementations. IoT devices are considered an area of interest for the attackers that are especially looking to compromise internal networks or hijacking devices for DDoS scenarios, although the number of DDoS attacks started to see a decline in recent quarters. Compromised IoT devices are acting as pivots and backdoors inside internal networks. Vulnerabilities affecting popular products are the primary drive when it comes to malware attacks on IoT devices, especially vulnerabilities that are affecting base software components. One of the 
www.conferenceie.ase.ro

important considerations regarding an IoT product it's the necessity of being connected to other products in order to fully benefit from the entire experience. Most IoT products are functioning offline and standalone however, important functionalities and features are usually linked to some form of connectivity to the internet and with other local devices. That interconnectivity between endpoints can expose components and networks. There are also privacy concerns linked to IoT products that are non-related to attacks and exploits. Sensitive client data manipulation, third party processing and the general data protection play and important when it comes to client's trust and security. Functionalities and capabilities are dictating, in this case, the sensitivity and the importance of product security and data privacy. Functionalities such as video and audio recording hold the biggest attention due to the nature of processed information. We see a significant increase in popularity, market value and share as IoT products are becoming a big part of our environment. This surge is directly linked with the number of threats that are showing an advance, pointing that security levels should be prioritized accordingly as the technology increases even further.

\section{References}

[1] A. Guzman and A. Gupta, IoT Penetration Testing Cookbook: Identify vulnerabilities and secure your smart devices. Packt, 2017.

[2] A. Gupta, The IoT Hacker's Handbook. Apress, 2019.

[3] S. Nicula. Robot hacking research. Internet: https://securitycafe.ro/2017/09/22/robothacking-research/, September 22, 2017 [Sept. 22, 2017].

[4] C. Cimpanu. New vulnerability lets attackers sniff or hijack VPN connections. Internet: https://www.zdnet.com/article/new-vulnerability-lets-attackers-sniff-or-hijack-vpnconnections/, December 05, 2019 [March 10, 2020].

[5] I. Adascalitei, "Smartphones and IoT Security," Informatica Economica, vol. 23, no. 2/2019, pp. 63-74, 2019.

[6] Shodan. Internet: https://www.shodan.io/, 2009 [Jan. 15, 2020].

[7] D. Demeter, M. Preuss and Y. Shmelev. IoT: a malware story. Internet: https://securelist.com/iot-a-malware-story/94451/, October 15, 2019 [Feb. 01, 2020].

[8] C. Cimpanu. Researcher: Backdoor mechanism still active in many IoT products. Internet: https://www.zdnet.com/article/researcher-backdoor-mechanism-still-active-in-many-iotproducts/, February 04, 2020 [Feb. 04, 2020].

[9] D. Bradbury. Why Successful IoT Bug Bounties Are So Rare. Internet: https://www.infosecurity-magazine.com/infosec/why-successful-iot-bug-bounties/, October 02, 2019 [Dec. 21, 2019].

[10] A. Clim, "Cyber Security Beyond the Industry 4.0 Era. A Short Review on a Few Technological Promises", Informatica Economica, vol. 23, no. 2/2019, pp. 34-44, 2019.

[11] HackerOne. 2019 Hacker Report. - HackerOne. Internet: https://www.hackerone.com/sites/default/files/2019-02/the-2019-hacker-report_3.pdf, August 21, 2019 [Aug. 22, 2019].

[12] Avira. Avira and TP-Link Join Forces to Offer Wi-Fi Routers with IoT Security for the Smart Home. Internet: https://www.avira.com/en/press/avira-and-tp-link-join-forces-tooffer-wi-fi-routers-with-iot-security-for-the-smart-home, September 17, 2019 [Sep. 17, 2019]. 